\title{
Violencia directa y conflictos distributivos sobre el agua. Evolución del debate analítico- conceptual y propuesta de nuevo enfoque
}

\author{
RAFAEL GRASA*
}

\section{RESUMEN}

El artículo busca dos objetivos: a) exponer, en clave comparativa y treinta años más tarde del momento inicial y candente, los debates sobre la relación entre conflictos armados o violencia directa y situaciones de estrés/ carencia hídrica o enfrentamientos distributivos (recursos o cuencas compartidas, por ejemplo) por el agua. Al hacerlo, se añadirán a la sistematización los nuevos y más recientes enfoques; y b) a partir de los resultados del punto anterior, proponer nuevas herramientas para el análisis y la intervención en dichos conflictos, aprovechando, a modo de ejemplo y no de análisis detallado, diez casos heterogéneos activos en el presente de conflictos distributivos con el agua como incompatibilidad crucial.

Los temas mencionados se popularizaron en los años noventa del siglo XX con afirmaciones impactantes que sostenían que "las próximas guerras no serían por petróleo o recursos minerales sino por el acceso al agua potable". En ese sentido, la comparación se centra en examinar el debate entre 1985 y 1995, donde el tema dominante fue si la escasez/concurrencia por el agua podía o no ser factor causal directo de conflictos violentos o si era simplemente un factor multiplicador o acelerador vinculado a otras causas, con las aportaciones y enfoques actuales. Estas aportaciones, frente a la omnipresencia en los años noventa de enfoques y textos basados en estudios de seguridad y la entonces llamada "seguridad ecológica", se centran actualmente en las herramientas que proporcionan los estudios sobre análisis, resolución y transformación de conflictos y en nuevas reflexiones epistemológicas respetuosas del sesgo cultural y de género y no occidentalocéntricas.

El texto, además de introducción y conclusiones, tiene tres apartados. El primero establece un punto de partida fáctico, resumiendo los datos relativos al agua dulce y potable, para establecer, mediante una sucinta descripción de diez conflictos, internos e internacionales, de varios continentes, una tesis central: la conflictividad más caliente, incluyendo conductas violentas, no es tanto por la carencia de agua en sí, sino por la gestión y gobernanza del agua, en general y en casos de escases de la misma. En segundo lugar, se resume las claves analíticas y los resultados del debate sobre los conflictos hídricos, en particular sobre los vínculos entre uso y escasez de agua y violencia directa desde finales de los años ochenta a mediados de los años noventa del siglo pasado. La tercera sección presenta nuevas miradas, basadas no en los estudios de seguridad o la seguridad ecológica, como antes, sino en los trabajos, consolidados, de la investigación para la paz y los estudios sobre análisis y transformación de conflictos. Concretamente, se exponen las aportaciones, y se propone como aplicarlas a los conflictos hídricos, mediante tres asunciones y siete tesis. Finalmente, las conclusiones insisten en la necesidad de tratar estos conflictos como conflictos sociales, mostrando que su especificidad está vinculada a la gestión y gobernanza del recurso más que a la escasez en sí y, al hacerlo, ponen el acento, en las dificultades de gobernabilidad y gobernanza que hay que abordar.

Palabras clave

Agua; conflicto social distributivo; violencia directa; intervención; gobernanza.

\section{Tithe}

Direct violence and distributive conflicts about water.The evolution of analytical debate and the proposal of a new approach

DOI:

https://doi.org// 0.15366/relacionesinternacionales2020.45.002

Formato de citación recomendado:

GRASA, Rafael (2020). "Violencia directa y conflictos distributivos sobre el agua. Evolución del debate analíticoconceptual y propuesta de nuevo enfoque", Relaciones Internacionales, $n^{\circ} 45$, pp. 53-7I.
* Rafael GRASA

Profesor titular de Universidad de Relaciones Internacionales en la Universitat Autònoma de Barcelona. Especializado en análisis y transformación de conflictos, en seguridad humana y reforma del sector de seguridad, teoría internacional

y desarrollo. Coordina el Máster

Universitario en "Relaciones Internacionales, Seguridad y Desarrollo" y el programa de Doctorado "Politics, Policies and International Relations" en la UAB. Contacto: rafael. grasa@uab.cat

Recibido:

31/05/2020

Aceptado:

10/10/2020 


\section{Abstract}

The article pursues two objectives: a) to comparatively trace the debates on the relationship between armed conflicts or direct violence and situations of stress / water shortage or distributive confrontations (resources or shared basins, for example) surrounding water, and in doing so add to the systematization of recent approaches; and b) based on the results of the aforementioned, propose new tools for the analysis and intervention in these conflicts, taking advantage, by way of example and not of detailed analysis, of ten current and heterogeneous cases of distributive conflicts over water.

This issue became popular in the nineties of the twentieth century with striking statements that proclaimed: "the next wars would not be for oil or mineral resources but for access to drinking water." In this sense, the current paper focuses on examining the debate between 1985 and 1995, where the dominant issue was on whether water scarcity or water competition could be a direct causal factor of violent conflicts, or if it was simply a multiplier or accelerating factor linked to other causes. These contributions, while previously dominated by the omnipresence of approaches and texts based on security studies and the then so called "ecological security", are now based on contributions from studies of the analysis, resolution and transformation of conflicts. Meanwhile, new epistemological reflections respectful of cultural and gender bias are also present, along with non-western-centric approaches.

Thirty years after the end of the Cold War, factual changes, new narratives, and novel guidelines for analysis have urged scholars to revisit the debate on the present and future relationships between armed conflict -or direct violence- and the scarcity or competition for water resources. I have just mentioned factual changes, among which I will highlight the following: significant ongoing changes in geopolitics and geo-economics; changes in the location and nature of direct violence, given that $80 \%$ of homicide deaths in the world are not related to direct violence of a clearly political nature (that is, armed conflict and terrorism), something that is linked with the strong growth of private security actors (legal and illegal) and a partial loss of the state's monopoly on the massive instruments of violence; ongoing mutations in the nature and spread of power; a progressive de-westernization of the international system and of the ways of analyzing it (international theory); the emergence of climate change and the climate emergency agenda and, in addition, the pandemics linked to recurrent zoonosis processes; and, to close this list, and returning to the topic being studies in this issue of the journal, the central position that water occupies in the 2030 agenda and in the Sustainable Development Goals (SDGs). Hence, we need new tools that allow us to analyze and better understand these conflicts related to water, in general, and also their occurrences in the major geopolitical areas of the world in the coming decades (Asia,Africa, Latin America and the Caribbean).

Regarding the new narratives and analysis guidelines, the paper focuses on the new approaches in the area of analysis, resolution and transformation of conflicts that have emerged from the consensus on liberal peace, along with contributions from political ecology specialized in distributive environmental conflicts and environmental justice. Taking into account these new epistemological reflections, including those related to identity, is important as it allows one to focus on causality, and the tools that facilitate its analysis

In addition to its introduction and conclusions, this article has three sections. The first establishes a factual starting point, summarizing the data related to fresh drinking water. Through a succinct description of ten conflicts, both intra and international, and from various continents and regions, this establishes a central thesis: the most significant conflict causation factor is not so much the lack of water, but the management and governance of water in general and its shortages. Secondly, it summarizes the analysis and the results of the debate on water conflicts, in particular on the causation links between water use and scarcity and direct violence from the late 1980s to the mid-1990s of the last century.The third section presents new perspectives, based not on security studies or ecological security, as before, but on the consolidated works of peace research, and studies on conflict analysis and transformation. Specifically, the main contributions and findings are displayed, and, also, a seminal framework to apply them to water conflicts, through three assumptions and seven theses. Finally, the conclusions insist on the need to treat these conflicts as social conflicts, showing that their specificity is linked to the management and governance of the resource rather than the scarcity itself.

In the concluding remarks, the paper highlights that the most pertinent features of any analysis and intervention in environmental conflicts, especially those that are persistent and difficult to resolve (what American literature usually calls "intractable" conflicts) do not reside in the adjective "environmental", or "hydric", as the mainstream suggested in the first half of the 1990s. What is really meaningful is that they are social conflicts, basically distributive conflicts, and linked to the search for fairer solutions; that is, related to the allocation, use and / or management of environmental goods.

The specificity provided by the adjective "environmental" or "hydric" exists, of course, beyond its growing importance for the contextual reasons already mentioned -such as demographic pressure, climate change or pollution and destruction of resources. However, this does not justify a differentiated treatment for these conflicts, as was the case previously. They are social conflicts, and therefore marked by cultural and contextual differences. Therefore, this means that they must be analyzed and intervened upon, in order to manage, resolve and transform them, with the same tools and techniques used for all the social distributive conflicts. We can call them "environmental", or "hydric" if that is the case, but only if this means that we do not stop dealing with them as social conflicts. And moreover, we must not forget that like most of these conflicts, it is necessary to apply "analytical filters" to them based on knowledge of the logic of collective action, public goods and collective goods

Finally, the paper argues that these conflicts and the new analytical and resolutions framework force us to modify the famous formula of "thinking globally, acting locally". Today, to tackle them, you also have to think in a multidimensional way, and, in many cases, act globally. In doing so, the paper emphasizes the governance and governance challenges that need to be addressed.

\section{KEYWORDS}

Water; social distributive conflict; direct violence; intervention; governance. 


\section{ntroducción}

El texto pretende revisitar los estudios sobre la relación causal, mediata o inmediata, que existe entre violencia directa y conflictos donde al agua es la contradicción, problema o incompatibilidad principal.Adicionalmente, se propone, de forma genérica y a partir de diez casos heterogéneos, una nueva manera de enfocar su análisis y la intervención en los mismos con ánimo de manejarlos, resolverlos o transformarlos.

Es decir, se busca reformular, treinta años más tarde y con nuevos enfoques, el debate sobre la relación entre conflictos armados y estrés/escasez hídrica o recursos/ cuencas compartidas, un tema que en los años noventa se popularizó con múltiples afirmaciones que sostenían que "las próximas guerras no serían por petróleo o recursos minerales sino por el acceso al agua potable". Concretamente, se reconstruye y compara el debate entre 1985 y 1995 -donde el tema dominante fue si la escasez/concurrencia por el agua podía o no ser factor causal directo de conflictos violentos o, por el contrario, sólo podía actuar como factor multiplicador o acelerador siempre y cuando existieran otras causas de conflictividad violenta o elementos contextuales decisivos- con otros enfoques y aportaciones actuales, que han abandonado ese sesgo dominante en los años ochenta y noventa. Estas aportaciones, frente a la omnipresencia en el primer período considerado de enfoques y textos basados en estudios de seguridad y la entonces llamada "seguridad ecológica", se fundamentan ahora, por el contrario, en las aportaciones y herramientas de los estudios sobre análisis, resolución y transformación de conflictos y en nuevas reflexiones epistemológicas respetuosas del sesgo cultural y de género y no occidentalocéntricas, como, por ejemplo, el fructífero enfoque del grupo "Waterscapes in Transcultural Perspective"!.

Lo cierto es que, como expondremos, treinta años después del fin de la guerra fría, cambios fácticos y nuevas narrativas y pautas de análisis aconsejan revisitar con nuevos marcos de análisis y explicaciones, como programa de investigación incipiente, el debate sobre la relación presente y futura entre conflictos armados/ocurrencia de violencia directa y escasez/competencia por recursos hídricos. Los cambios fácticos que acabo de mencionar son, sin pretensión de exhaustividad, los siguientes:

I) Cambios importantes en curso en la geopolítica y la geoeconomía; cambios en la ubicación y naturaleza de la violencia directa, habida cuenta de que el $80 \%$ de las muertes por homicidio en el mundo no están relacionadas con violencia directa de naturaleza claramente política (es decir, conflictos armados y terrorismo²).

2) Mutaciones en curso en la naturaleza y difusión del poder.

3) Una progresiva desoccidentalización del sistema internacional y de las formas de analizarlo (teoría internacional).

4) La irrupción de la agenda del cambio y de la emergencia climática y, además, las pandemias vinculadas a recurrentes procesos de zoonosis.

5) Y, para cerrar esta lista, y volviendo al tema que articular el presente número de la revista, la posición central que el agua ocupa en la agenda 2030 y en los Objetivos de Desarrollo

Ubicado en la Universidad de Heidelberg, que ha trabajado con múltiples ejemplos procedentes de Asia. Véase el clúster de excelencia de dicha universidad conocido como "Asia and Europe in a Global Context" y la serie de publicaciones bajo el título de Transcultural Research que edita Springer.

2 Algo que va unido al fuerte crecimiento de los actores privados de seguridad (legales e ilegales) y a una pérdida parcial del monopolio de los medios masivos de violencia por parte del estado. 
Sostenible (ODS).

De ahí que necesitemos nuevas herramientas y marcos analíticos que permitan entender mejor dichos conflictos vinculados al agua, en general, y también en sus ocurrencias en las grandes zonas geopolíticas del mundo en las próximas décadas (Asia, África, América Latina y el Caribe). Concretamente, prestaré atención especial a nuevos enfoques en el área de análisis y transformación de conflictos estratégicos que se han emancipado del consenso sobre la paz liberal, por un lado, y aportaciones de la ecología política especializada en conflictos ambientales distributivos y justicia ambiental, por otro. Un ejemplo de lo que digo: pese al interés inevitable que ofrece para el tema la cronología y la base de datos más usada en los últimos años, la del Pacific Institute, cuyo cofundador y director hasta 2016 fue Peter Gleick, me parece más importante centrarse en la causalidad y en las herramientas que permiten analizarla teniendo en cuenta esas nuevas reflexiones epistemológicas, incluyendo las vinculadas a temas identitarios (Kalpakian, 2017). De ello nos ocuparemos luego.

Para finalizar, he de decir que el empeño que acabo de enunciar y que abre una serie de trabajos futuros ya en curso, es a la vez una manera de revisitarme, puesto que se trata de un tema del que me ocupé bastante en la primera mitad de los años noventa, en el marco de trabajos individuales y colectivos del grupo de relaciones internacionales de mi universidad (Grasa, 1994, 1995, 1998, 2000).

En cuanto a la estructura, el texto está dividido en los siguientes apartados. Me ocuparé, en primer lugar, de establecer un punto de partida fáctico, resumiendo los datos relativos al agua dulce y potable, para establecer, mediante una sucinta descripción de diez conflictos, internos e internacionales, de varios continentes, una tesis central: la conflictividad más caliente, incluyendo conductas violentas, no es tanto por la carencia de agua, sino por la gestión y gobernanza del agua en general y de las mencionadas situaciones o contextos de escasez o carencia de la misma. En segundo lugar, se resume las claves analíticas y los resultados del debate sobre los conflictos hídricos, en particular sobre los vínculos entre uso y escasez de agua y violencia directa desde finales de los años ochenta a mediados de los años noventa del siglo pasado. La tercera sección presenta nuevas miradas, basada no en los estudios de seguridad o la seguridad ecológica, como antes, sino en los trabajos, consolidados, de la investigación para la paz y los estudios sobre análisis y transformación de conflictos. Concretamente, se exponen las aportaciones, y se propone como aplicarlas a los conflictos hídricos, mediante tres asunciones y siete tesis. Finalmente, las conclusiones insisten en la necesidad de tratar estos conflictos como conflictos sociales, mostrando que su especificidad está vinculada a la gestión y gobernanza del recurso más que a la escasez en sí.

\section{El punto de partida: la creciente centralidad del agua en la agenda internacional}

Empecemos con los datos desnudos. El agua es un requisito imprescindible para la vida y, aunque como es sabido la superficie con agua en nuestro planeta es mucho más importante que la terrestre, del total disponible de agua sólo un $2,5 \% \mathrm{~s}$ agua potable; el resto es agua salada situada en mares, océanos y otros espacios. Pero, si seguimos analizando, de ese $2,5 \%$ total, gran parte plantea dificultades de extracción: un $30 \%$ está en el subsuelo, un $68 \%$ en glaciares y zonas heladas; por tanto, sólo un I,2\% del $2,5 \%$ se encuentra en la superficie, y en lugares muy heterogéneos 
como los seres vivos, la atmósfera, los pantanos, las marismas y zonas húmedas, ríos y lagos y en el suelo helado y zonas de "permafrost" (Gleick, 1993). En suma, sólo un I/I50 del total de agua disponible en el planeta está en la superficie y, de ella, los seres humanos usamos básicamente para la vida cotidiana la que se encuentra en ríos y lagos (aproximadamente un $21 \%$ del total del agua potable en superficie se encuentra en lagos y aproximadamente un $0,5 \%$ en ríos). Si además tomamos en consideración que muchos de los ríos importantes del planeta, en todas las regiones, son cuencas que recorren muchos países y por tanto con numerosos estados ribereños, y que, además, en dichas cuencas se crean embalses y pantanos, tendremos una clara descripción de los riesgos de disputas de diversa índole.

Ello significa que, aunque el agua es un recurso renovable y en principio suficiente para todos los habitantes del planeta, el agua potable, en su mayor parte utilizada a partir de los ríos y lagos (lo que incluye, naturalmente, los embalses y pantanos artificiales para almacenarla), es un bien esencial del que no siempre las comunidades humanas pueden disponer en el momento oportuno, en el lugar oportuno y con la calidad necesaria. De hecho, la disponibilidad de agua dulce y potable siempre ha tenido un componente estacional y vinculado a la climatología. Por consiguiente, ha habido, hay y habrá situaciones de estrés hídrico, situaciones de escasez (de importancia diversa). De ello ser deriva, por tanto, que siempre han existido y existirán condiciones para que la diferente disponibilidad del recurso devenga motivo de incompatibilidad entre seres y grupos humanos, $y$, por tanto, base de disputas vinculadas a su posesión, control, gestión y uso, $y$, por ello, factor motriz de situaciones de conflicto (sea este interno, internacionalizado o internacional). En cualquier caso, conviene recordar que el deseo de disponer de agua (sea por necesidad, agravio, privación relativa o por ambición) es un rasgo habitual. Dicho de otra forma, el interés por el agua es antiguo y crecientemente importante entre grupos humanos dentro y fuera de las fronteras estatales. Como, también lo es la existencia dilatada de numerosos ejemplos de gestión cooperativa de las disputas y de acuerdos y tratados de diferente naturaleza; en suma, a buenas prácticas y lecciones aprendidas.

Concretamente, los trabajos del Pacific Institute — con Peter Gleick al frente- muestran que tal situación es antigua, muy antigua. De hecho, en sus cronologías y trabajos acumulativos, cita conflictos de hace tres mil y cuatro mil años, en particular en Oriente Medio y Próximo3. No obstante, como ya he señalado antes, esos datos y el panorama que acabo de describir desde hace más de una década está siendo objeto de nuevas miradas y narrativas, así como al impacto de otros factores causales.

Lo novedoso, en el momento en que se escribe el presente texto es que la demanda local y global de recurso hídricos no deja de aumentar a causa del crecimiento demográfico, del proceso de urbanización y de litoralización (ya vive en ciudades más del cincuenta por ciento de la población mundial y pronto llegará al sesenta por ciento) y de la creciente mejora de los ingresos globales de la población y su mayor consumo de recursos. Además, el cambio climático, cada vez más inevitable, y otros problemas de degradación medioambiental, incrementan e incrementarán las tensiones, habida cuenta de que están alterando de forma significativa en los próximos años

\footnotetext{
Sus cronologías y bases de datos proporcionan datos esenciales: fechas, partes o actores primarios implicados, disputa o antagonismo central en el conflicto (desarrollo, religión, uso o blanco militar, terrorismo, desarrollo, etcétera), una breve descripción de cada caso y, finalmente, las fuentes de las que proceden los datos reseñados.
} 
la disponibilidad estacional y regional y la calidad del agua, con impacto en el consumo humano y en la actividad productiva que consume gran parte del agua disponible, la agricultura. La razón principal es el deshielo parcial de los casquetes polares y el aumento del nivel del mar, merced al incremento de la temperatura a causa de la creciente acumulación de gases de efecto invernadero en nuestra atmósfera, que causará de forma generalizada, pero con impactos locales y regionales muy diversos, un aumento de los fenómenos meteorológicos extremos (inundaciones, sequías) y la desaparición o salinización de deltas importantes. Todo ello generará además un significativo incremento de la población desplazada y refugiada por razones medioambientales. En conjunto, podemos constatar la existencia de una creciente competencia por el agua $y$, por tanto, mayores riesgos de conductas violentas en los conflictos, en particular distributivos derivados de ello.

Todo ello genera preguntas importantes: a) ¿de qué conflictos estamos hablando, en el presente y en un futuro cercano?; b) dada la enorme heterogeneidad de conflictos con incompatibilidades vinculadas al agua, ¿se pueden usar pautas analíticas y explicativas comunes?

Para responder a las preguntas presentaré brevemente (por razones de economía expositiva y constricción de espacio) diez conflictos, de naturaleza y ubicación muy diferente, pero todos ellos con posibilidad y probabilidad de violencia directa. Si bien no se analizan con detalle en el presente artículo, servirán para presentar una propuesta de pauta de análisis, que se expondrá luego, y, en el futuro, serán objeto de aplicación detallada de la misma.A partir de estos ejemplos, plantearé una tesis respecto de la posibilidad de conductas violentas en todos ellos. Veamos pues esos diez heterogéneos casos elegidos para justificar la necesidad de una nueva pauta de análisis.

El primero es un clásico: la disputa por el agua en la cuenca del Nilo, centrada en el acceso y derechos sobre el agua entre los once países ribereños. Se trata de unos de los ejemplos más analizados históricamente, con interesantes experiencias de cooperación como la Iniciativa de la Cuenca del Nilo, con éxitos entre 1999 y 2007, aunque a partir de entonces se agravaron las disputas entre Egipto, Sudán y Etiopía, a causa de proyectos de presas, y la Iniciativa se sustituyó por negociaciones tripartitas. El segundo tiene que ver con las disputas en la cuenca del Mekong, también vinculado a grandes proyectos y construcciones de presas, sobre todo en China y Laos. Las tensiones han menudeado y la Comisión del Río Mekong ha tenido poca eficacia en la gestión de las mismas, por su escaso poder real y por la reticencia de China a ser y comportarse como miembro pleno de la misma. Ha preferido, como en otros casos, crear instrumentos ad hoc de cooperación con los estados ribereños de la parte baja de la cuenca. El tercero tiene que ver con las disputas por aguas transfronterizas entre Afganistán e Irán, en particular vinculadas al intento del primer país de usar las aguas de los ríos Helmand y Harirud en procesos de reconstrucción y de desarrollo postconflicto armado (en particular, presas e incremento de la superficie agrícola), que generaron alarma y tensiones con Irán, sobre todo por el escaso interés afgano en emprender negociaciones serias. El cuarto ejemplo es otro caso clásico, con miles de años de antecedentes antes de que surgieran los estados actuales: las disputas respecto de los ríos Tigris-Éufrates entre Turquía, Siria e Irak, en el que también interviene a veces Irán al ser parte de la cuenca del Tigris. Desde los años sesenta del siglo pasado diferentes proyectos unilaterales de irrigación que, junto a las tensiones políticas habituales, han provocado muchas tensiones, aunque existen plataformas e iniciativas de negociación desde hace un par de décadas. 
El quinto ejemplo, con lecciones interesantes de cooperación y de buenas prácticas, son las disputas sobre aguas transfronterizas entre Turquía y Armenia, que, como he dicho, han proporcionado buenos resultados de gestión. El sexto es un ejemplo de disputa interna, agravada recientemente por la abundancia de sequías: la disputa por el uso del agua de la cuenca del Cauvery entre los estados Karnataka y Tamil Nadu en la India, que ha derivado en protestas violentas en función de las decisiones y propuestas de los dos estados de alterar, sin acuerdo entre ambas, el flujo de agua del río y cuenca. El séptimo tiene que ver con otra disputa interna, un ejemplo emblemático de muchas semejantes en América Latina en los últimos años: las luchas y enfrentamientos violentos, con muertos, que se generaron en Cochabamba (Bolivia) a partir del año 2000 a causa de la privatización del agua de consumo doméstico. Lo interesante es que la vuelta a titularidad pública y el nuevo ordenamiento jurídico de la gestión del agua que surgió de las luchas no ha acabado con la que se llamó "guerra del agua", a raíz del sobreconsumo, deficiencias tecnológicas y de calidad y, en suma, de creciente carencia de suministro.

El octavo ejemplo, de gran interés por la conflictividad violenta y la intervención extranjera en el país actualmente, son las disputas ya antiguas en Yemen por la disminución de la disponibilidad de agua y la privación relativa y diferenciada entre la población, agudizada por la corrupción, el nepotismo, y más recientemente, el mencionado contexto de conflicto armado. El noveno elegido es la compleja relación entre sequía y escasez de agua, precios de los productos derivados de la agricultura y la ganadería, sin olvidar el conflicto armado, que se observa en Somalia. Los vínculos causales son complejos y difíciles de reconstruir, pero lo cierto es que están generando actividades ilícitas, problemas de subsistencia en las comunidades pastoriles, y, finalmente, incentivos significativos para que los afectos se añadan a grupos guerrilleros como Al Shabaab, como mostró la gran sequía de $20 \mathrm{I}$ I. El décimo y último ejemplo tiene que ver de nuevo con Egipto, pero en este caso es de naturaleza interna y no internacional. Hace años que el uso de recursos hídricos en Egipto supera claramente sus recursos internos renovables demostrados en gran medida vinculados a las aportaciones del Nilo. El crecimiento de la población, el incremento del consumo y el impacto del cambio climático están agravando una carencia o estrés estructural que puede incidir en la economía del país y serias restricciones para el consumo del país, sin olvidar, el impacto indirecto que ello tendrían en el problema —internacional- de la gestión de los recursos de la cuenca del Nilo con los otros estados ribereños.

Para concluir este apartado, plantearé una tesis central que deriva de la heterogeneidad y naturaleza diversa de los diez ejemplos de disputas sobre el agua que he resumido sucintamente. Soy consciente de que cada uno de ellos amerita, y exige, un análisis detallado, que como he dicho haré en el futuro partiendo de la pauta de análisis que luego presentaré. Pero al no disponer de lugar para hacerlo, me limitaré a presentar el resultado de dichos análisis como tesis a confirmar con estudios de caso.

Lo cierto es que todos esos conflictos, elegidos por seguir activos y ser muy heterogéneos, muestran que las tensiones distributivas por el uso del agua, por razones diferentes, no dejan de incrementarse y con ello también el riesgo de conductas violentas y, que, en muchos casos, no existen instrumentos adecuados, formales o informales, para negociar las disputas y controlar el riesgo de conductas violentas. 
De ahí la tesis que planteo, derivada de los diez conflictos variados que he descrito sucintamente: hay evidencia clara, en el momento actual que el riesgo de polarización, de escalada y de aparición de conductas violentas en disputas tan diferentes, y situadas en contextos y regiones también muy diversas, no es tanto la carencia de agua, sino la gestión y gobernanza de la misma. Dicho de otra forma, lo fundamental para el análisis y la intervención, como muestran esos diez casos, es que el riesgo de conflictividad violenta no depende tanto de la carencia de agua, aunque es un requisito inicial, sino de la capacidad de manejarla, de la gobernanza del conflicto entre los diversos actores, tanto si son internos como internacionales.

Si ello es así, como ahora veremos, resulta conveniente proponer un cambio importante en la forma de analizar la conflictividad vinculada al agua y, también, de intervenir en el manejo y transformación de los motivos de la disputa y en la prevención y tratamiento de las conductas violentas en los mismos. Para ello recordaremos brevemente las tesis e ideas dominantes entre finales de los años ochenta y mediados y finales de los años noventa, para luego, en el tercer apartado, centrarnos en la propuesta de nuevas pautas y herramientas.

\section{El debate sobre los conflictos hídricos en los años ochenta y mediados de los no- venta: claves y resultados}

Como ya he señalado, la preocupación por los conflictos vinculados al agua como recurso en disputa tiene una presencia antigua en la agenda, pero se incrementó en proporción geométrica a medida que desde, los años setenta (1972, Conferencia de Estocolmo), los temas medioambientales fueron ganando importancia en la agenda internacional. Los veinte años que van de Estocolmo a Río de Janeiro (1992, Conferencia sobre Medio Ambiente y Desarrollo), suponen la aparición de la preocupación por los límites de la biosfera a partir del informe de los Meadows para el Club de Roma, agenda de problemas globales medioambientales (lluvia ácida, reducción de la capa de ozono estratosférica, cambio climático, deforestación, pérdida de biodiversidad), el impacto del Informe Brundtland (1987) y la aparición del concepto de desarrollo sostenible o sustentable. En ese contexto, con una fuerte centralidad en la fijación de narrativas y de ideas de los estudios sobre seguridad (inspirados en el constructivismo, como en el caso de la Escuela de Copenhague, o en la teoría crítica, con un papel destacado de Ken Booth) y de la seguridad ecológica, los temas de seguridad medioambiental ganan relevancia y con ello ${ }^{4}$, la preocupación por la relación entre agua y conflictos con conductas de violencia directa se dio con particular relevancia entre mediados de los años ochenta y mediados de los años noventa.

Todo ello generó un debate enormemente importante, tanto en el eje de las grandes ideas y asunciones ontológicas (el conocido como eje temático), como, sobre todo, en los dos ejes más cercanos a la realidad fáctica, el eje analítico (programas de investigación y herramientas vinculadas, como bases de datos) y el eje fenoménico, el focalizado justamente en explicaciones causales empíricas. Dejaré de lado los debates en el eje temático y en el analítico para centrarme básicamente en lo ocurrido en el eje fenoménico. En éste, entre 1985 y 1995, gran parte del debate se dedicó justamente a explorar la vinculación causal entre violencia directa y factores ambientales, entre los cuáles tuvo un papel fundamental el de conflictos con el agua como incompatibilidad central. Me ocupé de todo ello — es decir, de la seguridad ecológica y de la relación entre conflictos

\footnotetext{
4 Para una explicación detallada, véanse Grasa, 2000 (capítulo con Ignacy Sachs) y Grasa 200 I.
} 
ambientales, entre ellos el agua, y la violencia directa - en textos anteriores, y no volveré sobre ello más que de forma sucinta. Sin ánimo de exhaustividad, lo hice, por ejemplo, en las siguientes aportaciones: caracterizando lo que denominé "conflictos verdes" internos e internacionales (Grasa, 1994), de forma general; aplicando las propuestas de Homer-Dixon (I991; Homer-Dixon, Boutwell y Rathjens, I 993) al caso del Mediterráneo (Grasa, I 995);y coordinando e introduciendo una sección monográfica sobre el tema en Ecología Politica ${ }^{5}$ (número I5; Grasa, 1998).

El resultado final de todo ese debate seminal puso en el centro a autores, $y$ en algunos casos a proyectos de investigación, que han seguido siendo fundamentales como referentes una o dos décadas después para dilucidar el grado de existencia o inexistencia de vínculos causales claros entre conflictos con incompatibilidades o motivos de disputa medioambientales (incluyendo el agua) y conductas de violencia directa por parte de los actores primarios implicados en dicha disputa.

Concretamente, destacaré, como fundamentales, cinco de ellos: a) Peter H. Gleick, y el trabajo colectivo bajo su dirección en el Pacific Institute ${ }^{7}$ (Gleick, 1993; Gleick, 1998-2018); b) el ya citado de Thomas Homer-Dixon y sus trabajos mencionados en el párrafo anterior; c) Thomas Naff, uno de los primeros que se ocupó, con un enfoque fuertemente neorrealista, de los conflictos hídricos ya en los años ochenta, en particular en Oriente Medio, con herramientas que permitían "calcular" matrices de poder (Naff y Martson, 1984); d) el proyecto Encop (Environment and Conflicts Project) en particular el trabajo recopilatorio de Kurt R.Spillmann y Günther Bachler (Spillmann, K.R y Bächler, G., 1995), así como los posteriores que han seguido ocupándose de la relación entre problemas medioambientales y conflictos violentos (potenciales o reales), así como los instrumentos para fomentar su manejo y resolución pacífica; y e) el trabajo, referente indiscutible desde hace décadas, de Miriam R. Lowi sobre la cuenca del río Jordán, que somete a contraste aproximaciones y pautas analítico-explicativas de matriz realista y de matriz liberal (Lowi, 1995).

Aunque el tratamiento del tema de la causalidad, es decir del eventual vínculo entre factores ambientales como incompatibilidad fundamental y conductas violentas en los conflictos, tiene tratamiento diferente en los cinco proyectos/trabajos que he elegido para plantear el estado del arte a mediados de los años noventa, se puede resumir la manera de abordar el problema diferenciando tres posiciones, de mayor a menor importancia dada a dicha vinculación:

1. Las que plantean una vinculación fuerte, directa, entre factores medioambientales y conductas de violencia directa, como en el caso de Gleick y Naff, ambos justamente basados en

\footnotetext{
5 El monográfico incluía, además de mi texto, un texto de Javier Sánchez sobre la evolución de la noción de seguridad y en concreto la "seguridad ecológica"; un texto de Geoffrey y David Dabelko recapitulando el debate y polémicas sobre seguridad medioambiental un texto de Nicole Gallant, que aterrizaba el debate en la oposición a un macroproyecto hidroeléctrico en Quebec; y dos artículos centrados en el tema del agua, uno de Donald Swain centrado en la escasez y estrés hídrico y uno de Ferran izquierdo sobre uno de los casos más estudiados en aquel momento, la cuenca del río Jordán (el otro, la cuenca del Nilo).

6 Cabe citar, adicionalmente, diversos prólogos y publicaciones de libros sobre el tema y, particularmente, el impulso a investigación sobre seguridad ecológica, conflictos ambientales y en concreto agua, tanto en el grado de ciencias ambientales como en el área de derecho público internacional y relaciones Internacionales. De ello surgirán diversos trabajos de Oriol Costa, por ejemplo, su trabajo final de grado en ciencias ambientales y luego su tesis doctoral, ya en relaciones Internacionales.

Pacific Institute for Studies in Development, Environment and Security (Database on Water and Conflict).

8 El texto citado recoge los trabajos de 60 expertos que, convocados por la Swiss Peace Foundation y el Center for Security Studies and Conflict Research (ETH) convocaron en octubre de 1994. Luego han seguido publicando textos importantes,
} 
conflictos hídricos. En ambos casos crearon mecanismos para aquilatar la vinculación antes del advenimiento de dichas conductas, índices o ratios en el caso de Gleick, matrices de poder en el caso de Naff. En cualquier caso, Naff es más rotundo que Gleick.

2. Las que sostienen que la vinculación puede existir, pero de darse suele ser mediada, indirecta, es decir, un factor acelerador o multiplicador, y que, además, su impacto depende en gran medida del contexto y de factores no medioambientales o no hídricos, como sostienen Homer-Dixon y la mayor parte de los resultados del proyecto Encop.

3. Los que no observan vinculación clara, al menos generalizable, ni siquiera en un caso concreto, como los conflictos, sincrónica y diacrónicamente considerados, presentes en la cuenca del Jordán, como sostiene Lowi.

En suma, el interés por los conflictos medioambientales y en particular los hídricos, desde mediados de los años ochenta a mediados de los noventa, muestra, como tendencia dominante, una serie de rasgos claros, que resumo a continuación. Primero, el interés sobre el tema se debió, sobre todo, a la creciente centralidad de los temas de medio ambiente en la agenda de relaciones internacionales y al impacto derivado de conciencia de los límites de la biosfera, que generó un aumento espectacular de la literatura dedicada a analizar la relación entre seguridad y medio ambiente, con un especial énfasis en la "lucha" por recursos escasos, renovables y no renovables. Segundo, una de las fuerzas motrices de ese acrecentado interés (así como de las expresiones con las que se aludía al mismo como "seguridad ecológica" o "seguridad medioambiental") fueron justamente los cambios en la concepción y la práctica de la seguridad que se dan entre finales de los años ochenta y mediados de los años noventa, en el marco de la posguerra fría.Tercero, las fuentes fundamentales de creación de pensamiento y de creación de agenda investigadora se encuentran en autores que proceden del campo en surgimiento de los estudios de seguridad, de académicos de Relaciones Internacionales y de estudios sobre desarrollo y medio ambiente, con escasa presencia de autores que proceden del campo del análisis, resolución y transformación de conflictos. Cuarto rasgo y último, ello explica, en gran medida, el hecho de que muchos autores plantearan análisis de causalidad cuasi ad hoc para los conflictos con incompatibilidades basadas en el agua, se tendiera a sobrevalorar la causalidad de la mera escasez de agua como factor de tensiones y eventual violencia directa frente a la gestión y gobernanza de la misma. En suma, ello explica que se dejaran de lado análisis de causalidad que prestan más atención a la sinergia e interrelación entre factores causales, es decir que dan especial importancia a la presencia de diversas incompatibilidades al mismo tiempo en una situación de conflicto y a la trascendencia de las posiciones, intereses y necesidades de los actores implicados.

Esa ausencia, o poca presencia, como expondremos, es justamente lo que aparecerá en los nuevos enfoques y tendencias. En efecto, hoy observamos, como se va a exponer en el siguiente apartado, la centralidad en el análisis de los conflictos hídricos de las herramientas analíticas y conceptuales de los estudios y práctica del análisis y transformación de conflictos. 


\section{Nuevas miradas, pautas y herramientas: las aportaciones del análisis, resolución y transformación de conflictos}

Empezaremos recordando que el campo del análisis y de la resolución de conflictos ${ }^{9}$ surgió del empeño de un grupo de pioneros multidisciplinares que consideraron útil estudiar el conflicto en tanto que fenómeno general, con características y rasgos compartidos en áreas tan diversas como las relaciones internacionales, las relaciones industriales, la política interna y las diversas incompatibilidades presentes en áreas tan diversas como naciones, comunidades, familias o individuos. Y, desde luego, compartiendo el enfoque seminal introducido en los años cincuenta por Lewis Coser: el conflicto, entendido como disputa y no como conducta violenta, es inevitable al ser un producto de las relaciones sociales, rasgo definitorio de la especie humana en virtud del hecho de ser un animal que vive agrupado, en ciudades, por citar el sentido original de la afirmación de Aristóteles ${ }^{10}$; e, incluso, puede ser, si se maneja bien, un factor positivo de cambio y mejora.

Estos primeros académicos pretendían inicialmente aplicar a todos los conflictos, incluyendo los civiles y los internacionales, enfoques que se estaban desarrollando en el ámbito de las relaciones industriales, de las relaciones internacionales desde enfoques heterodoxos y en el marco de la mediación comunitaria. Al principio, su empeño tuvo poco éxito, por dos razones: porque la comunidad académica de las Relaciones Internacionales usaba sus propias categorías para la comprensión de los conflictos internacionales, totalmente diferente de las del resto de las ciencias sociales, desafortunadamente; $y$, en segundo lugar, porque, objetivamente, no era fácil conciliar en las instituciones académicas y en la práctica diplomática tradicional la combinación de análisis y práctica que propugnaban dichos pioneros, sin ofrecer resultados tangibles más allá de talleres de formación y propuestas que no se aplicaban.

La propuesta de dichos estudios coincide, y en parte se solapa, con la aparición y evolución de la investigación para la paz (peace research), un intento todavía más amplio de conciliar ciencia y conciencia (introducir conciencia en la ciencia y dotar de ciencia a la conciencia). La propuesta, surgida en los años cincuenta y deudora del riesgo de guerra nuclear total, propone usar la herramienta más poderosa desarrollada por el ser humano, el conocimiento científico, para conocer las causas de las guerras y saber cómo establecer las condiciones de la paz (Grasa, 1990; Grasa, 2010).

Ambos enfoques comparten varios rasgos distintivos metateóricos, además del cientifismo racionalista y el interés por enfoques cuantitativos. Concretamente, la preocupación normativa y la convicción de que las causas de los conflictos armados son problemas intelectuales, cognoscibles, y por ende resolubles en el terreno práctico. En suma, los abordan como problemas intelectuales y académicos que, una vez resueltos, permiten establecer soluciones prácticas, lo que explica el énfasis constante en la aplicabilidad. Pero, finalmente y, sobre todo, comparten una concepción del

\footnotetext{
La expresión "resolución de conflictos" es ambigua, en la medida en que significa cosas diferentes para diferentes autores. Eso tiene que ver con el cuádruple origen del campo (cuatro tradiciones, por así decirlo): los trabajos procedentes del área de la gestión empresarial y de organizaciones, y del desarrollo organizativo; las Relaciones Internacionales (con aportaciones procedentes de la corriente dominante pero sobre todo del pensamiento heterodoxo; la práctica de ciertos grupos del movimiento por la paz y de la investigación para la paz; y, por último, las aportaciones de lo que se ha dado en llamar "resolución alternativa de disputas" en el terreno de los litigios empresariales, civiles y laborales, como alternativa a su tratamiento judicial.

10 A menudo traducida de forma inexacta como "animal político", cuando literalmente decía "animal que vive en ciudades" (o sea, en las polei).
} 
conflicto como algo inevitable, propio de las relaciones sociales del ser humano, no necesariamente violento ni malo, en línea con el ya mencionado Lewis Coser: una disputa o enfrentamiento entre actores que creen tener objetivos incompatibles respecto de uno o varios de los objetivos en pugna. De esa forma rompían con la tradición de separar las relaciones internacionales del resto de las ciencias sociales, algo que la corriente dominante de las relaciones internacionales no hará de forma generalizada hasta finales de la década de los años ochenta del siglo $X X$.

\section{I.Éxito y difusión de propuestas}

A partir de mediados de los años ochenta, y en particular en los años noventa, la investigación para la paz y la resolución de conflictos tuvieron éxito, parcial al menos, en la difusión de los enfoques y métodos elaborados para el análisis y la intervención en conflictos (Grasa, 20 I0; Deutsch, Coleman y Marcus, 2006; Bercovith, Kremenyukl y Zartman, 2009), una poderosa pero heterogénea caja de herramientas conceptual. La consolidación de los estudios y prácticas sobre resolución/transformación de conflictos resulta evidente a través de su institucionalización (cátedras, revistas, manuales, estudios de posgrado) y de su impacto. Destacaremos dos cosas pertinentes para lo que nos ocupa.

Por un lado, un impacto concreto: hacer que las Relaciones Internacionales hayan dejado de analizar los conflictos internacionales como caso aparte, de manera que sus análisis son en general los habituales en las restantes ciencias sociales. Por otro, algunos resultados más generales, que pueden sintetizarse así. Primero, sostener que lo que sabemos de conflictos, con algunas diferencias (cláusula de ceteris paribus), puede aplicarse a todo conflicto social, aunque cambie el nivel en que se aborda (nivel micro, meso o macro) o el tipo de conducta que manifiestan los actores. Segundo, generar diversas teorías y modelos explicativos, que, poco a poco han ido convergiendo y han reforzado los enfoques de epistemología racionalista moderada, que parten de la convicción de que se puede explicar y elegir plausiblemente entre teorías, preservando la intersubjetividad y la parsimonia explicativa y que preservan la distinción entre neutralidad moral (imposible) y objetividad (difícil o incluso imposible, pero irrenunciable como empeño). Tercero, hacer que se conviertan en herramientas de uso corriente propuestas y conceptos de autores inicialmente heterodoxos, como Kelman, Burton, Dobb, Azar, Kriesberg, y de centros que desarrollan esas aproximaciones (a menudo denominadas resolución de conflictos interactiva) como Lancaster, Kent, Bradford, Maryland o Harvard"'.

Todo ello ha dado lugar a un sustrato conceptual, articulado en torno al trabajo de estudiosos y prácticos en resolución de conflictos, basado en tres asunciones de partida y en un consenso teórico-metodológico, que presentaremos mediante seis afirmaciones o tesis, todas ellas referidas a problemas como las herramientas conceptuales de análisis y el tratamiento de la causalidad, y que nos permitirá abordar los conflictos vinculados al agua, en particular los que derivan en violencia directa, de una manera diferente.

\subsection{Las aportaciones: tres asunciones de partida y un consenso teórico-metodológico} La primera asunción, nuclear y básica, se articula en torno a la concepción del conflicto como un tipo de interacción o de interrelación entre partes (actores primarios), con posiciones,

\footnotetext{
- Respecto de la influencia derivada de su papel en conflictos concretos, bastará con aludir al caso sudafricano, a Oriente Medio, a Irlanda del
} Norte y, algo más tarde, a ejemplos en África y en el sureste asiático. 
necesidades e intereses que perciben como contrapuestos, pero no como un tipo determinado de conducta, en particular violenta. El conflicto, por ende, puede generar diversas conductas, algunas de las cuáles pueden ser violentas en determinadas situaciones; sin embargo, el motivo de la disputa, la incompatibilidad, no es a priori un determinante de la conducta violenta, aunque, en clave comparativa, puede afirmarse que cada conflicto concreto es más o menos proclive a generar conductas violentas desde su fase inicial. La segunda asunción afirma que el conflicto, entendido como persecución de objetivos cuya consecución simultánea los actores implicados consideran incompatibles por responder a intereses mutuos contrapuestos, es connatural al ser humano y a su organización social y, por ello, se da en diversas dimensiones: intrapersonal, interpersonal, intragrupal, intergrupal, internacional y global o sistémica. La tercera asunción, clave, considera el conflicto y las situaciones de conflicto como procesos, es decir, fenómenos cambiantes y dinámicos. De ello se deriva que todo conflicto pasa por fases o conflictos, incluyendo escaladas y desescaladas hacia conductas violentas, así como la posibilidad que durante el conflicto evolucionen las actitudes, intereses, incompatibilidades y conductas presentes. Por consiguiente, en términos de causalidad, resulta crucial identificar y entender las causas aceleradoras y multiplicadoras que permiten entender porque se llega a una fase violenta ${ }^{12}$.

En este punto debemos introducir ciertas aclaraciones que se derivan de las anteriores asunciones. Los conflictos vinculados al estrés o carencia de agua son conflictos en que los motivos de incompatibilidad o de disputa entre los actores primarios en disputa están relacionados con el agua $y$, por ello, las partes formulan demandas, que perciben como incompatibles, relacionadas con el uso, manejo o control del agua. Ello explica que pueda decirse que el agua tiene un papel central en el conflicto, por razones diversas: "propiedad" o titularidad de los derechos de uso, reglas de gestión de cursos o cuencas de agua compartidas, estrés hídrico, construcción de grandes infraestructuras, incremento de la polarización y antagonismo entre partes por otra (s) incompatibilidad (es), que agrava una disputa sobre el agua, etcétera. Pero a menudo el agua es sobre todo un acelerador o "precipitador" de conductas violentas, pero en presencia de otras incompatibilidades.

De las tres asunciones se derivan también importantes consecuencias para el análisis y la intervención, en particular en términos de causalidad y de herramientas vinculadas a la misma. Esas consecuencias están claramente presentes en el mencionado consenso teórico-metodológico surgido durante los años noventa, tanto para el análisis de la conflictividad, como para la forma de intervenir en los conflictos para su gestión, resolución y transformación. Sintetizaremos dicho consenso, a efectos de brevedad, con una serie de tesis que están detrás de y fundamentan los nuevos enfoques. Esas tesis son la base de la nueva pauta de análisis que se propone.

Primera tesis: los conflictos son procesos complejos y con rasgos diferenciados en función de los diferentes niveles de la actividad humana en que se dan, pero pese a todo existen elementos de estructura y proceso compartidos que permiten analizarlos con una herramienta común. Esa herramienta propone fragmentar el análisis de conflicto mediante la distinción en — cada una de las fases del conflicto, y siguiendo la propuesta seminal de Johan Galtung (1969 y siguientes) entre cada uno de los vértices de un triángulo que define la situación conflictiva: a) actitudes de los actores (lo que incluye percepciones, posiciones, intereses, necesidades y el impacto en

12 Para el caso de conflictos hídricos, véase por ejemplo Conca, 2012. 
las mismas de las conductas de las fases anteriores); b) conducta de los actores; y c) motivo (o motivos) de la incompatibilidad o contradicción entre los actores o partes.

Por otro lado, al hablar de los actores, la herramienta distingue, en función de la proximidad real a la incompatibilidad, a las conductas de reacción (en particular las violentas) y las actitudes e intereses en juego entre actores primarios (directamente afectados), secundarios (con intereses claros y conductas indirectas), terciarios (con intereses y conductas indirectas más difusas), y así sucesivamente. Los actores, cuando se trata de grupos o colectivos, en modo alguno son conjuntos monolíticos, sino que, como ya se ha apuntado, resultará del todo necesario entender procesos de elitización, acumulación de poder y de captura de recursos, propios de la polarización social asociada a los conflictos violentos.

Segunda tesis: cada conflicto concreto debe analizarse buscando las oposiciones e interacciones que se dan en cada fase del mismo entre sus actores primarios. Al hacerlo, resulta crucial comparar los intereses y necesidades en competencia,así como las diferencias en las actitudes y/o de las identidades en juego, así como sus eventuales cambios en diferentes momentos. Cara a la resolución o intervención, también resulta clave, por su impacto en eventuales negociaciones o regateos, distinguir entre posiciones de las partes (demandas concretas), intereses y necesidades:las posiciones suelen ser engañosas y difíciles de negociar, al ser públicas y discursivas; las necesidades marcan las líneas rojas o condiciones imposibles de aceptar pese al proceso de regateo, es decir aquello a lo que una parte no puede renunciar en modo alguno en la negociación; los intereses, delimitan el espacio en que se debe buscar el acuerdo mutuo, resultados gana-gana, el espacio en que se construyen los acuerdos buscando satisfacer intereses de todas las partes.

Tercera tesis: en cada situación conflictiva se debe prestar atención al contexto y a la correlación de fuerzas, con el objeto de decidir si se trata de un conflicto simétrico o asimétrico, en función del poder que tiene cada una de las partes. En este sentido, por ejemplo, el agua es un factor bien diferente de otro que también ha generado mucho debate en la posguerra fría en cuanto a la relación entre conflictividad y violencia directa. Las diferencias son notorias y vale la pena recordarlas sucintamente. La religión, además de conformar pautas de conducta, forma parte de las estructuras cognitivas que contribuyen a la formación de identidades y conformación de actitudes, es decir, forman parte de "modelos comunes de expectativas, orientaciones emocionales y percepciones que tienen los actores en una situación de conflicto” (Mitchell, 1989, p. 28). Por ello, aunque son básicamente factores que influyen a los individuos, en muchos conflictos contemporáneos se ejercen presiones políticas y sociales muy fuertes para que las actitudes individuales, en particular las religiosas, se adecuen a las creencias del grupo. El agua, por el contrario, es un factor material, tangible, aunque sujeto a percepciones y por ende a manipulaciones. Dicho de otra forma, resulta más sencillo dilucidar qué hay de cierto, de falso o de poco claro en una afirmación al respecto. No obstante, algunas propuestas de las nuevas epistemologías insisten también en que los factores identitarios pueden estar también presentes en conflictos hídricos.

Lo anterior nos lleva a recordar una distinción ya clásica entre conflictos donde la incompatibilidad o motivo de disputa está centrada en la dimensión inmaterial (de valores) y aquellos en los que predomina la dimensión material, las necesidades tangibles. En los primeros intervienen motivaciones profundas como los valores o las pautas culturales, lo que según la 
teoría da origen a disputas más intensas, con mayor facilidad de devenir en conductas violentas y por ende más difíciles de resolver, mientras que los conflictos con incompatibilidades centradas en necesidades tangibles o materiales, resultan más fáciles de gestionar y solucionar gracias a la búsqueda de compromisos entre los intereses en pugna (negociación en función de intereses y no de posiciones). Pero de nuevo, no existe un estudio científico sistemático de las implicaciones de esta dualidad, pero, para el caso que nos ocupa, conflictos con incompatibilidades materiales y tangibles, existe suficiente evidencia que permite, siempre y cuando como veremos luego, se aborde de manera diferente el tema de la causalidad, analizar e intervenir en dichos conflictos de forma diferente.

Y a ello aluden — a la causalidad — las tres tesis siguientes, lo que nos permitirá mostrar cuándo el agua deja de ser causa necesaria para ser suficiente, y en algunos casos, eficiente.

Cuarta tesis: la causalidad es siempre compleja, nunca monocausal en los conflictos sociales concretos, incluyendo los relacionados con temas hídricos. Dicho de otra forma, hay evidencia demostrada de que en ningún caso y en ningún tipo de conflicto existe una única causa explicativa o incompatibilidad entre las partes, en particular en los conflictos internacionales o los internos internacionalizados. Eso sí, en cada fase del ciclo dinámico del conflicto, puede singularizarse un factor causal predominante. Se abre así una vía interesante para tratar conflictos con el agua, puesto que, al ser básico, como hemos dicho la gestión o gobernanza de la escasez que la propia escasez del recurso, permite establecer el impacto de otros factores, del orden político, social, e incluso cultural e identitario.

Quinta tesis: al analizar un conflicto en términos de causalidad hay que ser cuidadoso y sutil en los matices. Concretamente, hay que distinguir entre "orígenes" y "causas", entre causa "eficiente" y causa "permisiva", y, sobre todo, entre causa "necesaria" y causa "suficiente". Veámoslo con más detalle. Primero, los orígenes, es decir los factores contextuales, históricos, culturales, diacrónicos y acumulativos, aportan mucha información sobre una situación conflictiva, pero no deben confundirse con las causas, que son siempre sincrónicas o de temporalidad corta. Concretamente, autores como Paul Wehr (Wehr, 2019) han insistido en que, si nos centramos en las causas, la descripción básica de cualquier conflicto —al menos en cada una de sus fasesse puede exponer en una sola página, algo que se ha convertido en "axioma" indiscutido desde hace años. Segundo, que hay que distinguir entre causalidad que efectivamente genera efectos mediante acción directa, causa eficiente, y causalidad permisiva, hechos, contextos o situaciones que incentivan, bien obstaculizan, determinadas conductas.Y, tercero y último, hay que diferenciar entre causas estructurales, necesarias, sin las cuáles no hay disputa antagónica entre partes, y causas suficientes, aquéllas que, en determinadas situaciones, permiten explicar por qué el conflicto llega hasta la violencia directa. Nuevamente, la distinción entre causas necesarias y causas suficientes da luz sobre la mayoría de los conflictos hídricos: la escasez del recurso es condición necesaria, estructural, para generar una disputa y un proceso de polarización; pero es la causa o causas suficientes las que permiten explicar por qué en algunos casos no hay forma de lograr cooperación y en otros sí entre las partes enfrentadas, $y$, en particular, por qué a veces surge la violencia directa.

Sexta tesis: la causalidad exige también tomar en consideración una dimensión temporal, de vínculos a corto, medio y largo plazo. Por decirlo, siguiendo la propuesta de los tres tiempos 
históricos de Braudel y la escuela de los Annales, debemos distinguir entre: causas estructurales (necesarias, de largo plazo, muy vinculadas con las asimetrías, $y$, en general, pocas y constantes); causas multiplicadoras o aceleradoras (que exacerban las incompatibilidades o las percepciones de imposibilidad de satisfacer los intereses contrapuestos de los actores); $y$, por último, causas desencadenantes, gatillos o detonadores (es decir fenómenos que colman el vaso, que precipitan los acontecimientos). Los factores estructurales son claves para explicar las causas necesarias, imprescindibles, mientras que los aceleradores, además de los desencadenantes, ayudan a explicar las causas suficientes, las que explican la ocurrencia. En el caso de los conflictos con incompatibilidades vinculadas con el agua, las causas estructurales suelen ser varias, y algunas de ellas no siempre vinculadas con el agua. Y también, por la centralidad de los problemas de gobernanza y gestión, tienen un papel clave en el análisis, y aún más en la intervención y transformación, las causas aceleradoras y multiplicadores.

Séptima y última tesis, para analizar e intervenir en conflictos medioambientales distributivos, debemos tener en cuenta una especificidad que incentiva su dificultad de arreglo o manejo ${ }^{13}$, aunque es compartida — quizás de forma menos fuerte- por otros conflictos sociales: la trascendencia de los factores perceptivos, los marcos en la persistencia de la disputa, del conflicto (Lewicki, Gray y Elliott, 2003). Por decirlo claramente, los conflictos "intratables” se caracterizan porque, aunque se transformen los contextos, cambien los actores e incluso se modifiquen las arenas y espacios en que se dirime la disputa y la incompatibilidad, la percepción de antagonismo, de conflicto que polariza, persiste. En ese punto, los marcos interpretativos influyen fuertemente en la percepción de intransigencia o en la posibilidad de lograr entre las partes, afectando a las percepciones de los actores implicados en cuento al diagnóstico, a las prospectivas de evolución de la incompatibilidad y también a las soluciones aceptables ${ }^{14}$. Los marcos por tanto son cruciales para que persista o se resuelva la percepción de "intratabilidad", como muestra con claridad los conflictos medioambientales vinculados a contaminantes tóxicos, por ejemplo. Naturalmente, los marcos también son dinámicos y por tanto pueden alterarse, a mejor o a peor, mediante intervenciones y actuaciones intencionales.

De estas siete conclusiones se deriva, por tanto, un nuevo marco o pauta de análisis, que se aplicará en el futuro.

\section{Conclusión}

Así las cosas, las tres asunciones y las siete tesis en que he resumido el consenso teóricometodológico actual permiten establecer una clara agenda futura. Por otro lado, posibilitan establecer las preguntas de investigación, la agenda y las pautas de análisis que deben guiar, ya sin debates estériles y habiendo incorporado las utilísimas herramientas del análisis y transformación de conflictos. En suma, asunciones y tesis hacen posible emprender nuevos exámenes e investigaciones de los conflictos donde el agua sea el motivo, o uno de los motivos, centrales de la disputa de manera diferente, capaz de percibir bien las causalidades, $y$, por tanto, ofrecer insumos para proponer formas de intervención más útiles.

13 Lo que la literatura estadounidense suele denominar "intratabilidad".

14 Por decirlo con la expresión médica que popularizó Galtung como manera de abordar el análisis e intervención en conflictos: diagnóstico, pronóstico y terapia, 
La propuesta puede armarse a partir de la constatación de que, pese a que como se ha mostrado con los diez ejemplos sucintamente presentados en el apartado primero del texto, lo habitual en los conflictos con motivos hídricos es la heterogeneidad y la pluricausalidad, eso no es específico de este tipo de conflictos, sino rasgo constitutivo de todos los conflictos sociales, por lo que, las herramientas del análisis y transformación de conflictos permiten abordarlos con pautas y herramientas para el análisis y la intervención útiles para cualquier conflicto y, a la vez, capaces de aprehender la especificidad y las pautas culturales diferentes y propias de cada caso. Adicionalmente, permiten poner en el centro un hallazgo crucial de los enfoques recientes, a saber: que los conflictos sobre el agua tienen mucho más que ver con la gestión y gobernanza del recurso que con la escasez del mismo. De hecho, lo fundamental para entender e intervenir en esos conflictos, como se dijo al principio del texto, no es tanto el motivo de la disputa o incompatibilidad, vinculada a carencias o luchas por el agua, sino los problemas de gobernanza y gobernabilidad.

En resumen, lo que se ha propuesto a lo largo del artículo es que lo relevante para analizar e intervenir en los conflictos medioambientales, sobre todo los persistentes y difíciles de resolver (lo que la literatura estadounidense suele denominar conflictos "intratables"), no debe buscarse en rasgos relacionados con los adjetivos "ambiental”, "medioambiental”, o "hídrico”, como sugirió la corriente dominante en la primera mitad de los años noventa. No, ese era y es un enfoque poco productivo. Lo realmente relevante es que son conflictos sociales, básicamente conflictos distributivos y vinculados a la búsqueda de soluciones más justas, es decir, relacionados con la asignación, uso y/o gestión de bienes medioambientales.

Lo específico que aporta el adjetivo "medioambiental” o "hídrico" — más allá de su creciente importancia por las razones contextuales ya comentadas como la presión demográfica, el cambio climático o la contaminación y destrucción de recursos- existe, claro, pero no justifica un tratamiento diferenciado y herramientas especiales, como sucedió antes. Son conflictos sociales, y por ende marcados por diferencias culturales y contextuales, y por ello, deben ser analizados - y debe intervenirse en ellos para gestionarlos, resolverlos y transformarlos - con las mismas herramientas, técnicas que en el resto de los conflictos. Podemos llamarlos "medioambientales", o "hídricos" si ese es el caso, pero sin que eso signifique abandonar el tratamiento del mismo como conflicto social distributivo, $y$, por tanto, sin olvidar que como a la mayoría de esos conflictos hay que aplicarles "filtros" analíticos basados en la lógica de la acción colectiva, los bienes públicos y los bienes colectivos.

Sus especificidades, que comparten con otros conflictos de nuestras sociedades a principios del siglo XXI, se vinculan a rasgos como los siguientes:

a) Forman parte de la agenda de debate y preocupación política, social y económica, y, por tanto, susceptibles de "securitizarse" por parte de las élites, fuerzas de seguridad, opinión pública e incluso la sociedad civil.

b) Son muy dinámicos y multidimensionales, puesto que las cuestiones medioambientales, y por tanto lo relativo al agua, se vinculan a temas de desarrollo, de bienestar y de seguridad, lo que complejiza su manejo y resolución, como demuestra el inacabado e inacabable debate que generó la noción de desarrollo sostenible. 
c) Son susceptibles de generar polarización, e incluso, de convertirse en marcos interpretativos de otros problemas, operando como creadores de marcos de identidad o de caracterización.

d) Plantean siempre problemas de gobernabilidad, gestión y gobernanza.

En suma,son conflictos, por su naturaleza social y los rasgos comentados como especificidad, que no pueden resolverse con simples soluciones técnicas, aunque puedan ayudar como condición necesaria, sino que requieran soluciones, opciones, políticas, decisiones que implican prioridades, coherencia entre diversas políticas públicas. Por eso las herramientas - ya muy consolidadasdel análisis, resolución y transformación de conflictos son las adecuadas.

Podríamos decir que estos conflictos nos obligan a modificar la célebre fórmula del “pensar globalmente, actuar localmente"; en la actualidad, para abordarlos hay también que pensar multidimensionalmente, $y$, en muchos casos, actuar globalmente. $Y$ eso es justamente lo que el autor abordará en próximos trabajos.

Todo lo expuesto anteriormente, empero, no quiere menoscabar la importancia geopolítica del agua y de los conflictos a ella vinculados. Valgan como recordatorio las certeras palabras del poeta,W.H.Auden: “miles de personas han sobrevivido sin amor, ninguna sin agua".

\section{Referencias}

Bercovitch, J, Kremenyuk, V. y Zartman,W. (2009). The Sage Handbook of Conflict Resolution. Londres: Sage.

Conca, K. (2012). Decoupling Water and Violent Conflict. Issues in Science and Technology, 29 (I), 39-48.

Deutsch, M, Coleman, P.T y Marcus, E.C (2006). The Handbook of Conflict Resolution. Theory and Practice. San Francisco: Jossey-Bass.

Gleick. P. H. (Ed.) (1993). Water in Crisis: A Guide to the World's Fresh Water Resources. Nueva York: Oxford University Press.

Gleick, P. H (I998-20II). The World's Water $1998-1999$ (Volume I): The Biennial Report on Freshwater Resources. Washington: Island Press. Se han consultado también los seis volúmenes posteriores, hasta 20II, editados también por Island Press, y el 7 y 8 (20I3, 2018), publicados por el Pacific Institute de California.

Grasa, R. (1994). Los conflictos “verdes”: su dimensión interna e internacional. Ecología Política, 8, 25-40.

Grasa, R. (1995). El Mediterráneo desde una perspectiva globalizadora de la seguridad. Una mirada a la dimensión cooperativa de la conflictividad. Papers, 46, 25-42.

Grasa, R. (1998). Las nuevas concepciones de la seguridad: el debate sobre la seguridad ecológica o seguridad ambiental. Ecología Política, I5, 7-10.

Grasa, R y Ulied, A. (Eds.) (2000). Medio ambiente y gobernabilidad. Diagnóstico y sostenibilidad en el Mediteráneo. Barcelona: Icaria.Véase especialmente: Grasa, R. y Sachs, I. (2000). Ecodesarrollo y sostenibilidad: sugerencias para la aplicación de nuevas estrategias de desarrollo, 9|-126.

Grasa, R. (200 I). La construcción de regímenes internacionales para la protección transfronteriza del medio ambiente y de los recursos naturales: ¿sistemas de gobernación o sistemas de gobierno? Papeles y Memorias de la Real Academia de Ciencias Morales y Políticas, I0, I I2-133.

Grasa, R. (20l0). Cincuenta años de evolución de la investigación para la paz.Tendencias y propuestas para observar, investigar y actuar. Barcelona: Generalitat de Catalunya.

Homer-Dixon, T. (I99I). On the Threshold. Environmental Changes as Causes of Acute Conflict. International Security, $\mathrm{XVI}, 2,76-\mathrm{II} 6$

Homer-Dixon,T., Boutwell,J.H.y Rathjens, G.W.(1993). Environmental Change andViolent Conflict. Growing scarcities of renewable resources can contribute to social instability and civil strife. Scientific American, febrero, 38-45.

Kalpakian, J (2017). Identity, Conflict and Cooperation in International River Systems, Londres: Routledge.

Lewicki, R.J., Gray, B. y Elliott, M. (2003). Making Sense of Intractable Environmental Conflicts. Concepts and Cases. Washington: Island Press.

Lowi, M.L. (1995). Water and Power. The Politics of a scarce resource in the Jordan River basin. Cambridge: Cambridge University Press. ( $2^{\circ}$ edición, que actualiza la inicial de 1993).

Mitchell, C.R. (1989). The Structure of International Conflict. Nueva York: St. Martin's Press.

Naf, T. y Matson, R. (Eds.) (1984). Water in the Middle East: Conflict or Cooperation? Boulder:Westview Press. 
Spillmann, K.R.y Bächler, G. (Eds.) (1995). Environmental Crisis:Regional Conflicts andWays of Cooperation. Berna / Zúrich: Encop Papers.

Wehr, P. (2019). Conflict Regulation. Londres:Taylor \& Francis. 


\section{RELACIONES INTERNACIONALES}

Revista académica cuatrimestral de publicación electrónica

Grupo de Estudios de Relaciones Internacionales (GERI)

Universidad Autónoma de Madrid, España

https://revistas.uam.es/relacionesinternacionales

ISSN 1699 - 3950

f facebook.com/RelacionesInternacionales

3. twitter.com/RRInternacional 